This information is current as of April 26, 2023.

\title{
Acute Life-Threatening Hemorrhage in Patients with Head and Neck Cancer Presenting with Carotid Blowout Syndrome: Follow-Up Results after Initial Hemostasis with Covered-Stent Placement
}

H. Shah, J.J. Gemmete, N. Chaudhary, A.S. Pandey and S.A. Ansari

AJNR Am J Neuroradiol 2011, 32 (4) 743-747

doi: https://doi.org/10.3174/ajnr.A2379

http://www.ajnr.org/content/32/4/743 


\section{ORIGINAL RESEARCH}

\section{H. Shah}

J.J. Gemmete

N. Chaudhary

A.S. Pandey

S.A. Ansari

\section{Acute Life-Threatening Hemorrhage in Patients with Head and Neck Cancer Presenting with Carotid Blowout Syndrome: Follow-Up Results after Initial Hemostasis with Covered-Stent Placement}

\begin{abstract}
BACKGROUND AND PURPOSE: CSP in patients with HNC presenting with CBS can provide immediate hemostasis to prevent exsanguination. We evaluated the safety and efficacy of CSP to control acute life-threatening hemorrhage in patients with HNC presenting with CBS.
\end{abstract}

\begin{abstract}
MATERIALS AND METHODS: We retrospectively reviewed 10 patients $(7$ men, 3 women; mean age, 59 years) with HNC presenting with acute life-threatening hemorrhage from CBS that was treated with CSP. We studied patient demographics, presentations, procedures, initial and delayed complications, and technical and clinical outcomes on follow-up.
\end{abstract}

RESULTS: All patients achieved immediate hemostasis following CSP. Periprocedural complications consisted of groin hematomas $(n=2)$, acute limb ischemia requiring thrombectomy, and an asymptomatic temporal lobe hemorrhage. Imaging and clinical follow-up were available for a mean of 17.7 months (range, 1-60 months). Two patients remained asymptomatic with a patent stent and no evidence of rebleeding at 17 and 21 months, respectively. Recurrent hemorrhages requiring retreatment were encountered in 3 patients secondary to stent infections $(30 \%)$ at mean duration of 8 months. Neurologic morbidity resulted from stent thrombosis and stroke at 8 months in a single patient. Mortality was unrelated to CSP but was a result of palliative hospice care $(n=3)$ at a mean of 2 months or natural disease progression $(n=1)$ with documented patency of the stent at 6 months.

CONCLUSIONS: Acute life-threatening hemorrhage from CBS related to advanced HNC can be safely and effectively treated with CSP. However, potential delayed ischemic or infectious complications are common in the exposed or infected neck.

\begin{abstract}
ABBREVIATIONS: $A C T=$ activated clotting time; $A P=$ anteroposterior; $A S A=$ acetylsalicylic acid; $\mathrm{CA}=$ cancer; $\mathrm{CBS}=$ carotid blowout syndrome; $\mathrm{CCA}=$ common carotid artery; $\mathrm{CSP}=$ coveredstent placement; $C T x=$ chemotherapy; DSA = digital subtraction angiography; FFR = free flap reconstruction; $H N C=$ head and neck cancer; ICA = internal carotid artery; $L=$ left; $L C C A=$ left CCA; $L x=$ laryngectomy; PTFE = polytetrafluoroethylene; $P x=$ pharyngectomy; $R$ = right; RCCA = right $C C A ;$ RICA = right ICA; RND = radial neck dissection; $S C C$ = squamous cell cancer; $\mathrm{SND}=$ select neck dissection; $\mathrm{XRT}=$ radiation therapy.
\end{abstract}

c BS is a life-threatening complication associated with HNC and its therapy. ${ }^{1,2}$ The reported incidence of carotid artery rupture after radial neck dissection is $4.3 \% .{ }^{3}$ Patients with CBS can have a variety of presentations with the most significant being acute life-threatening hemorrhage. CBS more frequently occurs in patients with $\mathrm{HNC}$ and those with radiationinduced necrosis, recurrent tumors, wound complications from neck dissection, or vessel erosion from pharyngocutaneous fistulas. Emergency surgical management of CBS is often technically difficult to perform in a previously irradiated neck and is associated with a $40 \%$ and $60 \%$ neurologic morbidity

\section{Received April 17, 2010; accepted after revision September 3.}

From the University of Michigan Medical School (H.S.), Ann Arbor, Michigan; Division of Interventional Neuroradiology (J.J.G., N.C.) and Department of Neurosurgery (A.S.P.), University of Michigan Health System, Ann Arbor, Michigan; and Division of Interventional Neuroradiology (S.A.A.), University of Chicago, Chicago, Illinois.

Please address correspondence to Joseph J. Gemmete, MD, Division of Interventional Neuroradiology, University of Michigan Health System, B1D530 UH SPC 5030, 1500 E Medical Center Dr, Ann Arbor, Ml, 48109-5340; e-mail: gemmete@umich.edu

EIndicates article with supplemental on-line table.

DOI 10.3174/ajnr.A2379 and mortality rate, respectively. ${ }^{4}$ Deconstructive techniques, such as coil embolization and permanent balloon occlusion of the compromised vessel, have substantially improved the complication rate of CBS compared with surgical vessel exclusion. Zero percent to $15 \%$ of patients with CBS who are managed with permanent balloon occlusion may develop immediate or delayed cerebral ischemia as a consequence of $\geq 1$ factor, such as an incomplete circle of Willis, thromboembolism arising from an acutely occluded carotid artery, and delayed collateral failure. ${ }^{1,4-8}$

Given these results, there has been an interest in treating CBS with the deployment of covered stents. Self-expanding covered stents reconstruct the vessel wall with relatively atraumatic deployment, excluding extraluminal vessel pathology and maintaining intraluminal blood flow to the intracranial circulation, thus possibly limiting ischemic complications and immediately controlling the hemorrhage. To date, there remain limited data on the use of covered stents for the treatment of CBS in patients with acute life-threatening hemorrhage in the setting of $\mathrm{HNC}$, with complication rates ranging between $0 \%$ and $27 \% .^{8-12}$ The purpose of this study was to 
evaluate the safety and efficacy of CSP in achieving immediate hemostasis in patients with acute life-threatening hemorrhage in the context of HNC presenting with CBS.

\section{Materials and Methods}

\section{Methods}

We retrospectively reviewed patient medical and imaging records for demographics, presentations, technical procedures, initial and delayed postprocedure complications, technical outcomes (or patency of the covered stents), and clinical outcomes. The institutional review boards at the University of Michigan Hospital, Ann Arbor, Michigan, and the University of Chicago, Chicago, Illinois, approved this study.

Specifically, we acquired data for patient age and sex, clinical presentations, prior surgical or medical treatments for SCC of the head and neck, initial site of the hemorrhage, condition of the local wound or infection, immediate angiographic and clinical results, postprocedural and delayed complications, the site and time interval of recurrent hemorrhage, follow-up imaging (Doppler sonography/DSA), and clinical results.

\section{Procedure}

Informed consent was obtained from the patient or legal representative for the use of a covered stent. Nearly all patients had imaging evaluation with CT angiography for initial diagnosis and preoperative planning (vascular access, pseudoaneurysm location, area of extravasation, and vessel diameter). All procedures were performed with the patient under general anesthesia to protect the patent airway, because all patients had significant hemorrhage at the time of presentation. Given this clinical scenario, we thought the quickest approach to resuscitation and stabilization of the patient hemorrhage was by CSP. Following induction of general anesthesia, cervical and cerebral DSA identified or confirmed the site of extravasation, vessel compromise, or pseudoaneurysm. The patients were emergently loaded with clopidogrel (Plavix), $600 \mathrm{mg}$, and ASA, $325 \mathrm{mg}$, via a nasogastric tube. One patient was loaded with abciximab (ReoPro), $0.25 \mathrm{mg} / \mathrm{kg}$, intravenously due to the absence of orogastric or nasogastric access secondary to massive gauze packing in the oral pharynx. All patients were fully anticoagulated with intravenous heparin to maintain an ACT of $>250$ seconds just before deployment of the stent.

Following selective catheterization of the CCA, a 300-cm Amplatz guidewire (Boston Scientific, Natick, Massachusetts) was advanced into the ICA/external carotid artery, and the existing sheath was exchanged for an 80-cm 9F sheath (Cook, Bloomington, Indiana), which was advanced into the proximal CCA or brachiocephalic artery. A self-expandable nitinol stent covered with PTFE (Fluency stent; Bard Peripheral Vascular, Tempe, Arizona) was deployed across the area of extravasation or pseudoaneurysm with $1 \mathrm{~cm}$ of coverage proximal and distal to the diseased segment. A proximal occlusion balloon was not placed if active extravasation was seen. Confirmation of occlusion of the area of extravasation or pseudoaneurysm was confirmed on immediate follow-up angiography. All patients were continued on ASA, $81 \mathrm{mg}$, and clopidogrel, $75 \mathrm{mg}$, for 12 weeks after CSP, followed by ASA, $81 \mathrm{mg}$, indefinitely.

\section{Results}

The patient population included 7 men and 3 women with a mean age of 59 years (age range, 45-75 years). All patients presented with a history of head and neck SCC status postsurgical neck dissections and radiation. Seven patients under- went a laryngectomy with 5 also requiring free flap reconstructions. The presentations varied, including neck stoma bleeding $(n=1)$, enlarging neck hematomas or pseudoaneurysms $(n=4)$, wound dehiscence after flap reconstruction with bleeding $(n=1)$, and oropharyngeal bleeding $(n=4)$. Nine patients presented with bleeding from a carotid artery, and 1 patient from a brachiocephalic artery pseudoaneurysm or area of active extravasation (Fig 1). The patients' clinical characteristics are summarized in the On-line Table.

Initial angiographic and clinical outcomes were successful in all 10 patients with deployment of 11 covered stents. Placement of a self-expandable PTFE-covered stent provided immediate hemostasis and reconstruction of the vessel wall, confirmed with postprocedural angiography in all cases. One patient required covered stents placed across each carotid bifurcation with tumor encasement. On angiography, the pseudoaneurysm or area of active extravasation was seen in the CCA $(n=6)$, CCA bifurcation $(n=4)$, and right brachiocephalic artery $(n=1)$. Placement of a covered stent (diameter, 7-10 mm; length, 40-60 $\mathrm{mm}$ ) was sufficient to achieve immediate hemostasis without the need for adjunctive techniques such as coil embolization of larger pseudoaneuryms.

Periprocedural complications were limited to groin hematomas $(n=2)$, acute limb ischemia in a patient with severe peripheral vascular disease treated successfully with a right common femoral artery cut-down and thrombectomy, and an asymptomatic $2-\mathrm{cm}$ right temporal lobe intraparenchymal hemorrhage related to an intravenous bolus of abciximab. There were no vessel dissections, pseudoaneurysm ruptures, or thromboembolic or ischemic complications.

Imaging and clinical follow-up were available for a mean 17.7 months (range, 1-60 months). There was no procedurerelated mortality, but overall mortality included 3 patients who were placed in palliative care and died in hospice at a mean duration of 2 months. Additionally, 1 patient died from natural HNC progression with documented patency of the stent at 6 months and no recurrent bleeding. Two patients have remained asymptomatic with a patent stent and no evidence of repeat hemorrhage at 17 and 21 months, respectively.

There was no immediate procedure-related morbidity for a mean duration of 9.7 months. However, delayed complications and morbidity occurred in 4 of 10 patients (40\%) related to either recurrent bleeding secondary to stent infection $(n=3)$ or stent thrombosis and stroke. Stent infection was associated with the development of a persistent carotid-pharyngocutaneous fistula in a single patient. A second patient had multiple cerebral abscesses from an infected stent, which were likely septic emboli (Fig 2). These were successfully treated with long-term antibiotics. The interval between the initial endovascular procedure and recurrent bleeding ranged from 3 to 15 months (mean, 8 months). Retreatment involved surgical removal of the infected covered stent in all 3 patients to eliminate the infective source. One patient presenting with a repeat CBS required a balloon test occlusion study and subsequent endovascular carotid sacrifice. In contrast, the 2 remaining patients underwent successful surgical bypass with a right carotid-subclavian bypass graft and a carotid interposition graft by using an explanted right superficial femoral artery. The manifestations of the recurrent bleeding episodes were 

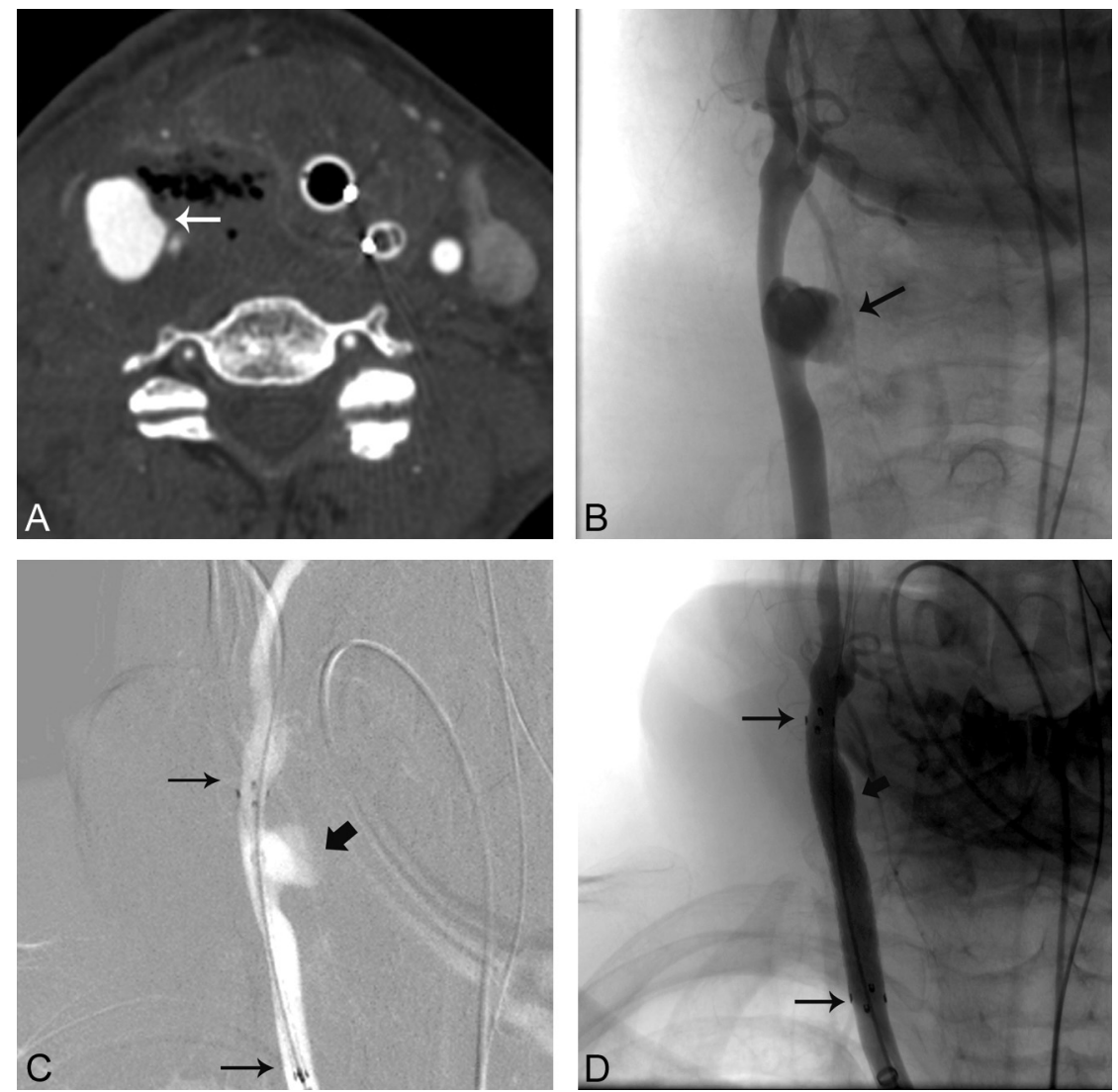

Fig 1. A 51-year-old woman with glottic and hypopharyngeal cancer who presented with hemoptysis (On-line Table). A, Axial contrast-enhanced CT scan of the neck shows extravasation from the RCCA (arrow) with surrounding air. B, AP RCCA angiogram shows the area of extravasation (arrow). C, AP roadmap of the RCCA shows placement of a covered stent (long arrows) across the area of extravasation (short arrow). D, AP RCCA angiogram after covered stent (long arrows) placement shows no evidence of extravasation (short arrow).

similar to those of the initial CBS presentations (On-line Table).

\section{Discussion}

CBS is a dreaded iatrogenic complication of HNC treatment. Since the syndrome was first described in 1962, several surgical and endovascular treatment options have been attempted. ${ }^{13}$ Before the advent of endovascular treatment modalities, CBS could be treated only with surgical ligation or surgical bypass of the carotid artery. However, open surgical techniques in the setting of HNC were associated with high mortality and major neurologic morbidity rates of $40 \%$ and $60 \%$, respectively. ${ }^{4}$ Endovascular management of acute CBS by balloon occlusion of the carotid artery was first introduced in $1984 .^{14}$ This treatment gained popularity because of the elegance and relative ease of the approach, especially in a hostile post-surgical or irradiated neck. Moreover, management with permanent balloon occlusion resulted in significantly lower morbidity and mortality rates than the surgical approach. ${ }^{1,4,6}$ Despite this advancement, there is a rate of $0 \%-15 \%$ of cerebral ischemic complications with permanent balloon occlusion of the ICA or CCA. ${ }^{1,4-8}$

The ischemic complications from vessel-deconstruction strategies subsequently led to the use of endovascular stents as a possible option. Many initial reports suggested that endovascular stents could not only reconstruct the arterial wall but also maintain vessel patency and decrease ischemic complications. Macdonald et $\mathrm{al}^{15}$ first reported a case of CBS managed successfully with CSP in an emergent presentation. Lesley et $\mathrm{al}^{11}$ published a series of 16 CBS cases treated with endovascular stents, including 2 covered stents, resulting in no deaths, and 1 transient ischemic event. Kim et al ${ }^{12}$ described a series of 4 CBS cases treated successfully with CSP without associated neurologic morbidity or mortality. In fact, these reports are similar to our findings because we noted no initial mortality or neurologic ischemic complications after CSP in our population. It follows that covered stents may represent an excellent treatment technique for the acute management of CBS, especially in patients who are at a high risk for stroke as a result of poor intracranial collaterals or an incomplete circle of Willis.

Early encouraging reports were mixed, with a series of complications associated with the use of covered stents. Warren et $\mathrm{al}^{9}$ reported 3 patients with CBS who were initially managed successfully with CSP, but on follow-up, 2 of these stents had extruded, leading to stent thrombosis in 1 patient and stroke in another. Simental et $\mathrm{al}^{10}$ similarly reported on delayed complications of stroke and stent thrombosis in 2 cases of CBS treated with CSP. Chang et $\mathrm{al}^{16}$ described a delayed case of brain abscess formation as a result of septic emboli from an infected self-expandable covered stent used for CBS. Chang et $\mathrm{al}^{17}$ also reported their experience in 24 patients with CBS who underwent endovascular therapy. Initial complications were encountered in 4 patients $(36.4 \%)$ who underwent reconstructive management and in 1 patient $(7.7 \%)$ who underwent deconstructive management $(P=.142)$. Rebleeding occurred in 5 patients $(45.5 \%)$ in the reconstructive management group 

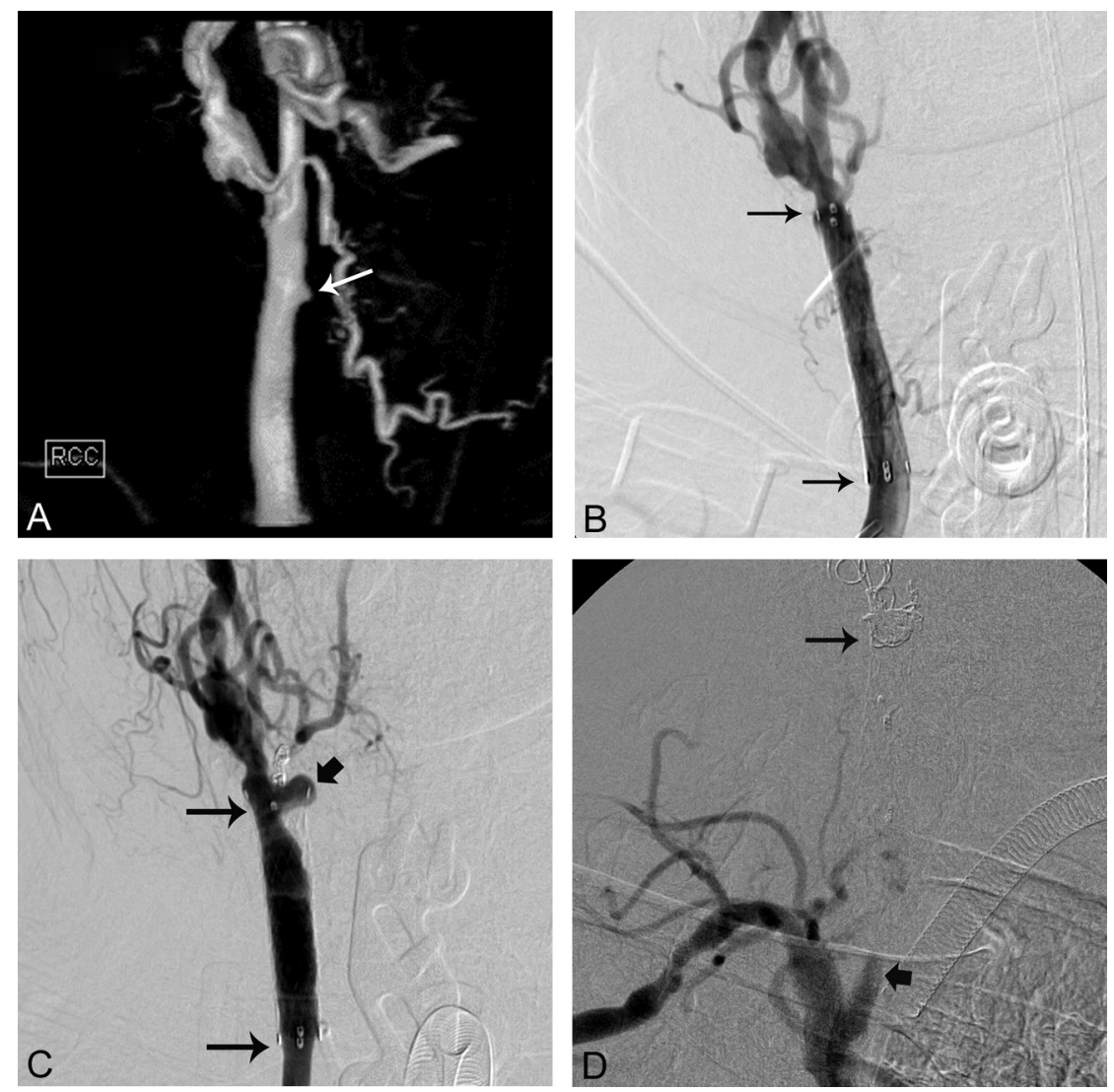

Fig 2. A 65-year-old man with laryngeal cancer who presented with bright red blood from the tracheostomy site (On-line Table). A, 3D surface-rendering angiogram of the RCCA shows a small pseudoaneurysm (arrow). B, AP RCCA angiogram shows placement of a covered stent (long arrows) across the pseudoaneurysm. C, Three-month follow-up RCCA angiogram shows the development of a pseudoaneurysm (thick arrow) at the distal end of the covered stent (long arrows). D, After coil occlusion of the RCCA and RICA, a right brachiocephalic arteriogram shows occlusion of the RCCA (thick arrow) with coils within the RCCA (long arrow).

and in 3 patients $(23.1 \%)$ in the deconstructive management group $(P=.659)$

In our series, 3 patients (30\%) had delayed complications of stent infections requiring stent extraction after endovascular carotid sacrifice or surgical bypass. In these cases, the stent may have become chronically infected after being deployed in an area of infective necrosis, serving as a nidus of infection and facilitating the formation of thrombi or septic emboli in the carotid artery. In fact, 1 patient developed multiple cerebral abscesses. ${ }^{18}$ Stent infection complications could not be entirely eliminated despite systematic prescription of postprocedural antibiotics.

We routinely initiate intravenous broad-spectrum antibiotics for 7-10 days postprocedure with adequate bacterial coverage native to the skin and oropharynx. Next, we place the patient on long-term antibiotic coverage with oral clindamycin, especially if there is the possibility of a chronically infected neck or dehiscent wound. Covered stents are prone to a high incidence of infection when deployed in an open environment, either communicating with the skin superficially or the deep aerodigestive tract. Most interesting, all 3 infected covered stents in our series exhibited communication with the aerodigestive tract and presented with recurrent hemorrhages. Pyun et al ${ }^{19}$ showed a recurrent bleeding rate in 6 of 7 patients treated with CSP within a short timeframe from 3 to 44 days. They postulated that the recurrent bleeding rate was most likely due to underlying infection or injury to the vasa vasorum after radiation therapy.
Recurrent hemorrhage is a feared complication of CSP and may be directly related to infection of the stent. Recurrence rates of $33 \%-86 \%$ have been quoted in prior studies. ${ }^{11,17,19,20}$ In comparison, our rebleeding rate was (3/10) 30\%. The recurrent hemorrhage rate in our series was slightly lower than that in other studies and may be due to our extended use of long-term antibiotics. All recurrences were managed successfully with either endovascular carotid sacrifice or surgical bypass followed by surgical debridement and stent extraction. Patients treated with endovascular covered stents must be cautioned on the possibility of rebleeding and followed closely for signs of recurrent CBS

We advocate CSP in the acute emergent setting of a patient with inadequate collateral circulation presenting with CBS. This is a straightforward procedure in experienced hands and can achieve immediate cessation of the hemorrhage with minimal risk of stroke. Primary vessel sacrifice in the acute emergent setting is still an excellent option in patients with good collateral circulation. It is possible to get control of the bleeding on the table with a temporary balloon before proceeding with permanent vessel sacrifice without the procedure being excessively time-consuming. Realistically, the number is too low in any of these series individually or in summation to form definitive conclusions about the risk of disabling neurologic events with 1 procedure versus the other, except in cases of inadequate collateral circulation, in which case CSP has an obvious advantage.

Once hemostasis is achieved, a balloon test occlusion fol- 
lowed by vessel sacrifice can be performed in a more controlled setting if infection develops in the region of the covered stent. The potential of infection in these patients is high due to associated open or dehiscent wounds and/or communication with the aerodigestive tract. Therefore, we suggest following these patients every 3-6 months with CT angiography of the neck or sonography to look for fluid or evidence of a pseudoaneurysm developing around the stent. In contrast, after permanent vessel sacrifice, there is very minimal risk of infection with recurrent delayed CBS.

To prevent thromboembolic complications related to stent placement, we routinely load patients with clopidogrel, 600 $\mathrm{mg}$, and ASA, $325 \mathrm{mg}$, through a nasogastric tube just before the procedure. Furthermore, aggressive heparin anticoagulation is used during the procedure with repetitive ACT monitoring. Patients remain on clopidogrel, $75 \mathrm{mg}$, and ASA, 81 $\mathrm{mg}$, daily postprocedure for at least 12 weeks. Following documentation of stent patency by Doppler sonography, the patient is placed on ASA, $81 \mathrm{mg}$ daily, for life. It is possible that our relatively low rate of ischemic complications resulted from a strict adherence to the use of dual antiplatelet therapy. In our study, only 1 patient could not be administered the dual antiplatelet regimen, and a loading intravenous dose of a glycoprotein IIb/IIIa inhibitor was substituted during stent placement. Unfortunately, this patient had an asymptomatic $2-\mathrm{cm}$ left temporal lobe intracranial hemorrhage, and antiplatelet agents (clopidogrel and ASA) were not initiated until 2 days postprocedure. The same patient had stent thrombosis and stroke at 8 months postprocedure, very likely coincidental. To prevent thromboembolic complications, we believe extended oral antiplatelet agents are mandatory, despite a possible increased risk of hemorrhagic complications. Parallel antiplatelet protocols have eloquently shown efficacy in the carotid and coronary stent literature. $^{21}$

Several limitations of our study are readily apparent. The retrospective analysis invariably produces internal bias, and the relatively small number of patients makes generalization to a large cohort of similar patients very difficult. Unfortunately, the pathology is so rare that any single institution cannot recruit enough patients for the statistical power that multicenter registries or meta-analyses may provide. All patients were treated with the same manufactured covered stent (Bard Fluency); therefore, a comparison regarding the type of covered stent graft that may be advantageous for the treatment of CBS is impossible. Furthermore, the importance of postprocedure management with dual antiplatelet regimens is assumed from the non-covered stent literature. The time required for PTFE endothelialization, its thrombogenicity, and for antiplatelets to prevent thromboembolic complications is largely unknown.

\section{Conclusions}

CBS related to advanced HNC can be safely and effectively managed with CSP in the acute emergent setting. However, potential delayed ischemic or infectious complications are common in the exposed or infected neck. After hemostasis is obtained, a temporary balloon occlusion test with possible deconstructive management should be considered if infection develops in the covered stent. Close follow-up and extended use of antiplatelet agents and broad-spectrum antibiotics may prevent some acute and chronic complications associated with CSP.

\section{References}

1. Chaloupka JC, Putman CM, Citardi MJ, et al. Endovascular therapy for the carotid blowout syndrome in head and neck surgical patients: diagnostic and managerial considerations. AJNR Am J Neuroradiol 1996;17:843-52

2. Wong SJ, Machtay M, Li Y. Locally recurrent, previously irradiated head and neck cancer: concurrent re-irradiation and chemotherapy, or chemotherapy alone? J Clin Oncol 2006;24:2653-58

3. Maran AG, Amin M, Wilson JA. Radical neck dissection: a 19-year experience. J Laryngol Otol 1989;103:760-64

4. Citardi MJ, Chaloupka JC, Son YH, et al. Management of carotid artery rupture by monitored endovascular therapeutic occlusion (1988-1994). Laryngoscope 1995; 105:1086-92

5. Dare AO, Chaloupka JC, Putman CM, et al. Failure of the hypotensive provocative test during temporary balloon test occlusion of the internal carotid artery to predict delayed hemodynamic ischemia after therapeutic carotid occlusion. Surg Neurol 1998;50:147-55, discussion 155-56

6. Chaloupka JC, Roth TC, Putman CM, et al. Recurrent carotid blowout syndrome: diagnostic and therapeutic challenges in a newly recognized subgroup of patients. AJNR Am J Neuroradiol 1999;20:1069-77

7. Adams GL, Madison M, Remley K, et al. Preoperative permanent balloon occlusion of internal carotid artery in patients with advanced head and neck squamous cell carcinoma. Laryngoscope 1999;109:460-66

8. Chang FC, Luo CB, Lirng JF, et al. Complications of carotid blowout syndrome in patients with head and neck cancers treated by covered stents. Interv $\mathrm{Neu}$ roradiol 2008;14 (suppl 2):29-33

9. Warren FM, Cohen JI, Nesbit GM, et al. Management of carotid "blowout" with endovascular stent grafts. Laryngoscope 2002;112:428-33

10. Simental A, Johnson JT, Horowitz M. Delayed complications of endovascular stenting for carotid blowout. Am J Otolaryngol 2003;24:417-19

11. Lesley WS, Chaloupka JC, Weigele JB, et al. Preliminary experience with endovascular reconstruction for the management of carotid blowout syndrome. AJNR Am J Neuroradiol 2003;24:975-81

12. Kim HS, Lee DH, Kim HJ, et al. Life-threatening common carotid artery blowout: rescue treatment with a newly designed self-expanding covered nitinol stent. Br J Radiol 2006;79:226-31

13. Borsanyi SJ. Rupture of the carotids following radical neck surgery in radiated patients. Eye Ear Nose Throat Mon 1962;41:531-33

14. Osguthorpe JD, Hungerford GD. Transarterial carotid occlusion: case report and review of the literature. Arch Otolaryngol 1984;110:694-96

15. Macdonald S, Gan J, McKay AJ, et al. Endovascular treatment of acute carotid blow-out syndrome. J Vasc Interv Radiol 2000;11:1184-88

16. Chang FC, Lirng JF, Tai SK, et al. Brain abscess formation: a delayed complication of carotid blowout syndrome treated by self-expandable stent-graft. AJNR Am J Neuroradiol 2006;27:1543-45

17. Chang FC, Lirng JF, Luo CB, et al. Patients with head and neck cancers and associated postirradiated carotid blowout syndrome: endovascular therapeutic methods and outcomes. J Vasc Surg 2008;47:936-45

18. Oweis Y, Gemmete JJ, Chaudhary N, et al. Delayed development of brain abscesses following stent-graft placement in a head and neck cancer patient presenting with carotid blowout syndrome. Cardiovasc Intervent Radiol 2011; 34(Suppl 2):S31-S35

19. Pyun HW, Lee DH, Yoo HM, et al. Placement of covered stents for carotid blowout in patients with head and neck cancer: follow-up results after rescue treatments. AJNR Am J Neuroradiol 2007;28:1594-98

20. Patsalides A, Fraser JF, Smith MJ, et al. Endovascular treatment of carotid blowout syndrome: who and how to treat. J NeuroIntervent Surg 2010;2:87-93

21. Fiorella D. Anti-thrombotic medications for the neurointerventionalist: aspirin and clopidogrel. J NeuroIntervent Surg 2010;2:44-49 